\title{
Genomic Profiling Maps Loss of Heterozygosity and Defines the Timing and Stage Dependence of Epigenetic and Genetic Events in Wilms' Tumors
}

\author{
Eric Yuan, ${ }^{1}$ Chi-Ming Li, ${ }^{1}$ Darrell J. Yamashiro, ${ }^{2}$ Jessica Kandel, ${ }^{3}$ Harshwardhan Thaker, ${ }^{4}$ \\ Vundavalli V. Murty, ${ }^{4}$ and Benjamin Tycko ${ }^{1,4}$ \\ ${ }^{1}$ Institute for Cancer Genetics; ${ }^{2}$ Department of Pediatrics, Division of Pediatric Oncology; \\ ${ }^{3}$ Department of Surgery, Division of Pediatric Surgery; and ${ }^{4}$ Department of Pathology, \\ Columbia University Medical Center, New York, New York
}

\begin{abstract}
To understand genetic and epigenetic pathways in Wilms' tumors, we carried out a genome scan for loss of heterozygosity (LOH) using Affymetrix 10K single nucleotide polymorphism (SNP) chips and supplemented the data with karyotype information. To score loss of imprinting (LOI) of the IGF2 gene, we assessed DNA methylation of the $H 195^{\prime}$ differentially methylated region (DMR). Few chromosomal regions other than band 11p13 (WT1) were lost in Wilms' tumors from Denys-Drash and Wilms' tumor-aniridia syndromes, whereas sporadic Wilms' tumors showed LOH of several regions, most frequently $11 \mathrm{p} 15$ but also $1 p, 4 q, 7 p, 11 q, 14 q, 16 q$, and 17p. LOI was common in the sporadic Wilms' tumors but absent in the syndromic cases. The SNP chips identified novel centers of $\mathrm{LOH}$ in the sporadic tumors, including a 2.4-Mb minimal region on chromosome 4q24-q25. Losses of chromosomes $1 p, 14 q, 16 q$, and 17p were more common in tumors presenting at an advanced stage; 11 p15 LOH was seen at all stages, whereas LOI was associated with early-stage presentation. Wilms' tumors with LOI often completely lacked LOH in the genome-wide analysis, and in some tumors with concomitant $16 \mathrm{q}$ LOH and LOI, the loss of chromosome 16q was mosaic, whereas the H19 DMR methylation was complete. These findings confirm molecular differences between sporadic and syndromic Wilms' tumors, define regions of recurrent $\mathrm{LOH}$, and indicate that gain of methylation at the H19 DMR is an early event in Wilms' tumorigenesis that is independent of chromosomal losses. The data further suggest a biological difference between sporadic Wilms' tumors with and without LOI. (Mol Cancer Res 2005;3(9):493-502)
\end{abstract}

Received 6/20/05; revised 8/18/05; accepted 8/19/05 Grant support: NIH grant R01 CA102270 (B. Tycko).

The costs of publication of this article were defrayed in part by the payment of page charges. This article must therefore be hereby marked advertisement in accordance with 18 U.S.C. Section 1734 solely to indicate this fact.

Requests for reprints: Benjamin Tycko, Division of Pathology, Columbia University Medical Center, 630 West 168th Street, New York, NY 10032. Phone: 212-305-1149; Fax: 212-305-5498. E-mail: bt12@columbia.edu Copyright (C) 2005 American Association for Cancer Research. doi:10.1158/1541-7786.MCR-05-0082

\section{Introduction}

Since 1972 when Knudson and Strong analyzed epidemiologic data from Wilms' tumor to develop the concept of tumor suppressor genes (1), research on this pediatric kidney tumor has continued to uncover important principles in cancer genetics. Early findings were the recognition of the Wilms' tumor-aniridia (WAGR) syndrome and its mapping to chromosome $11 \mathrm{p} 13$ (2-4) and the discovery of the WT1 tumor suppressor gene in that chromosome band (5-7). WT1 is mutated in $\sim 10 \%$ of Wilms' tumors overall, predominantly in the setting of WAGR and Denys-Drash syndromes but also in a small subset of sporadic Wilms' tumors (8-10).

WT1 mutations are rare in sporadic Wilms' tumors; instead, a second locus, called WT2 and mapping to chromosome $11 \mathrm{p} 15.5$, is the more common target of loss of heterozygosity (LOH). WT2 most likely corresponds to the closely linked and oppositely imprinted genes IGF2 and H19 in this chromosomal band. In the $\sim 45 \%$ of sporadic Wilms' tumors that have $11 \mathrm{p} 15.5 \mathrm{LOH}$, there is a selective loss of maternal alleles and duplication of paternal alleles (reviewed in ref. 11). This results in biallelic expression of IGF2 mRNA and loss of expression of H19 RNA. In another large group of sporadic Wilms' tumors, both parental alleles are retained, but the maternal allele of $\mathrm{H} 19$ and its upstream insulator sequences become hypermethylated at $\mathrm{CpG}$ sites, leading to the same end point of biallelic IGF2 expression [loss of imprinting (LOI)] and $H 19$ silencing. In the Beckwith-Wiedemann syndrome, a subset of affected individuals show gain of methylation at $H 19^{\text {mat }}$ and LOI of $I G F 2$ in their somatic tissues, leading to somatic overgrowth and a strong predisposition to Wilms' tumor (12). Recently, rare Beckwith-Wiedemann syndrome cases were identified with germ-line microdeletions in the H19 upstream sequences (13), a genetic finding that further supports the equivalence of $W T 2$ and $I G F 2 / H 19$.

In addition to chromosome band $11 \mathrm{p} 15.5$, cytogenetic and LOH studies have implicated several other chromosomal regions in sporadic Wilms' tumors. One copy of the q-arm of chromosome 16 is sometimes lost, typically via unbalanced chromosomal translocations (see ref. 14 and references therein). The resulting 16q hemizygosity correlates positively with tumor anaplasia $(14,15)$. Other chromosomes previously implicated by cytogenetics and/or LOH include losses and gains of portions of chromosome 1 (16), abnormalities of chromosome 7, notably $\mathrm{i}(7 \mathrm{p})$ in which the p-arm is lost and q-arm is reduplicated (17-21), and loss of chromosome 14q (22). To examine LOH at 
higher resolution and with complete genomic coverage and to gain insights into the relationship between genetic and epigenetic events in Wilms' tumors, we have carried out a genome-wide scan at high resolution in syndromic (WAGR or Denys-Drash) and sporadic Wilms' tumors using Affymetrix $10 \mathrm{~K}$ single nucleotide polymorphism (SNP) chips. The results of this scan, supplemented by DNA methylation and cytogenetic analyses, provide insights into the mechanism and timing of genetic and epigenetic events in Wilms' tumors.

\section{Results}

Genome-Wide Analysis of $\mathrm{LOH}$ in Sporadic and Syndromic Wilms' Tumors

We applied probes made from Wilms' tumor and normal kidney DNAs to Affymetrix 10K SNP chips. Allele call rates in our series ranged from $74.7 \%$ to $99.6 \%$, with the $74.7 \%$ call rate representing an outlier case (mean call rate, 94.9\%; median, $96.3 \%$ ). We first validated the SNP chip data by comparing the results for selected chromosomal regions with data from conventional microsatellite analysis, RFLP analysis, and DNA sequencing. We have genotyped previously many of the Wilms' tumors for a series of microsatellite and RFLP markers on chromosome 11p (23), and the LOH results for 25 of the 26 tumors included in both that previous study and the current one were concordant. The single discrepancy was accounted for by partial (mosaic) LOH, visible in microsatellite analysis but not detected by the SNP chips. Similar concordance was evident from comparing the current data with our prior microsatellite genotyping for markers on chromosome 16q (14), with a single discrepancy ( 1 of 25 cases), which was again accounted for by low-level mosaic $\mathrm{LOH}$, detected by microsatellite analysis but not by the SNP chip. Additional independent validations of the SNP chip data for a small region of reduction to hemizygosity on chromosome $4 \mathrm{q}$ and for $\mathrm{LOH}$ of markers on chromosome $7 \mathrm{p}$ are shown in subsequent sections.

Among the 62 sporadic Wilms' tumors, matched tumor and normal DNA samples were available for 46 cases, with unpaired tumor DNA available from the remaining 16 cases. The boundaries of regions of $\mathrm{LOH}$ could be ascertained at high resolution for the cases with paired samples (Fig. 1, blue bars), but the high density of SNPs on the microarrays also allowed detection of large regions of allelic loss in the unpaired cases (Fig. 1, green bars). Such regions, representing segments of DNA greater than $\sim 15 \mathrm{Mb}$, are detected as contiguous stretches of $>100$ SNPs giving uniformly homozygous genotypes (AA and BB). We assessed specificity and sensitivity of this method by reexamining the data for our paired tumor/
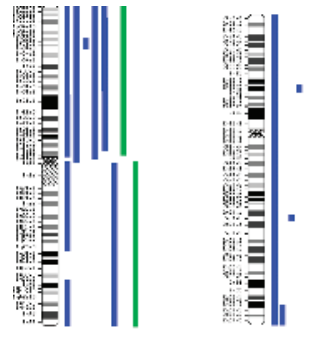

Chr 1

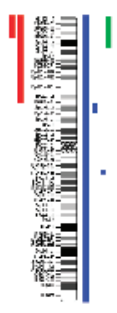

Chr 3

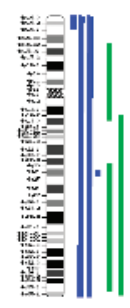

Chr 4
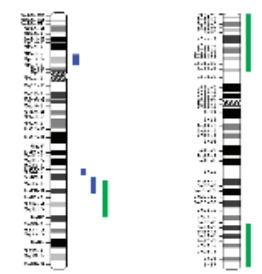

Chr 5

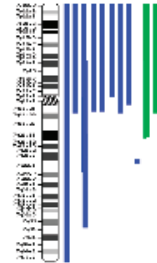

Chr 6

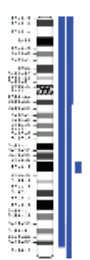

Chr 7
Chr 8
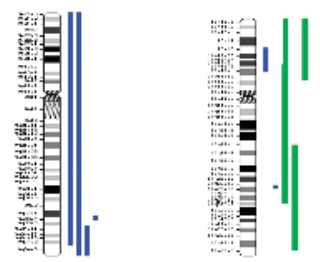

Chr 9

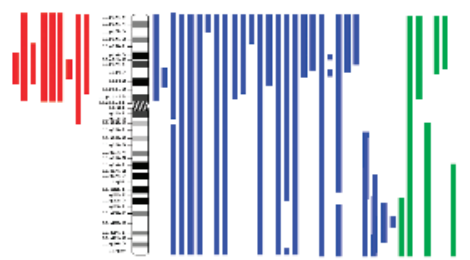

Chr 11

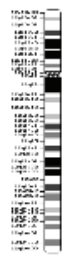

Chr 12

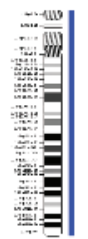

Chr 13

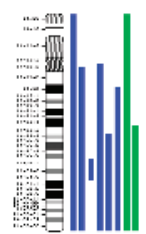

Chr 14

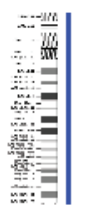

Chr 15

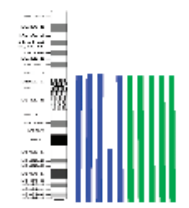

Chr 16

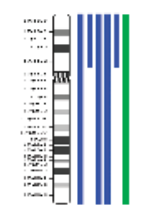

Chr 17

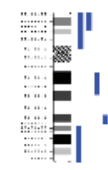

Chr 18
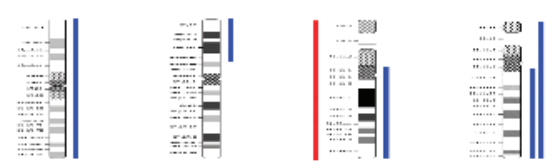

Chr 19

Chr 20

\section{Chr 21 Chr 22}

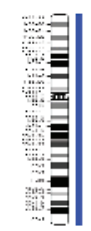

FIGURE 1. 10K SNP mapping of LOH in sporadic and syndromic Wilms' tumors. The regions of LOH, with the boundaries determined by markers that retain heterozygosity or by telomeres, are indicated by bars next to each chromosome. Red bars, data from syndromic Wilms' tumors; blue and green bars, data from sporadic Wilms' tumors; blue bars, regions of LOH determined from tumors with paired normal DNA; red and green bars, regions of LOH determined as contiguous stretches of $>100$ homozygous SNPs in tumors without paired normal DNA. 
normal cases, treating these tumors as unpaired. This procedure indicated a moderately high sensitivity for $\mathrm{LOH}(67.4 \%)$, with a high specificity (false-positive rate of $4.5 \%$ ) using this cutoff of $>100$ contiguous homozygous SNPs.

Examination of the euchromatic regions of all chromosome arms, except the Y-chromosome, revealed chromosome 11p as the most common site of allelic loss (Fig. 1). This finding is consistent with prior LOH mapping by us and others (23-25). These prior LOH surveys, and cytogenetics (26-29), have implied that Wilms' tumors are often genetically stable, and we therefore expected a low baseline rate of $\mathrm{LOH}$ on other chromosomes. As shown in Fig. 1, this baseline rate is in fact very low, with only 12 of the 41 euchromatic chromosome arms showing $\mathrm{LOH}$ at frequencies above $5 \%$. The most prominent "secondary peaks" of LOH were on chromosome arms 11q, 7p, $16 q, 14 q, 17 p$, and $1 p$, in that order of frequency, with an additional interesting region of recurrent $\mathrm{LOH}$ on $4 \mathrm{q}$ (Fig. 1). The LOH on chromosome arm 11q reflected loss of alleles along the entire chromosome in some cases, likely driven by selection for loss of the distal p-arm, but the mapping also indicated an independent region of $\mathrm{LOH}$ on the q-arm of this chromosome (Fig. 1). As discussed below, an unexpectedly large percentage of Wilms' tumors, $12(19 \%)$ sporadic cases in this series, showed no detectable LOH. Of these 12 cases, 9 were tumor/ normal pairs, and the conclusion that these tumors lacked $\mathrm{LOH}$ is therefore based on the full resolution of the $10 \mathrm{~K}$ chips.

In contrast to the findings in the sporadic Wilms' tumors, nine Wilms' tumors from WAGR or Denys-Drash syndromes did not show LOH in any of these specific secondary regions (Fig. 1). As predicted from prior work, these syndromic cases, which invariably contain WT1 mutations, showed stretches of homozygous SNPs (reflecting hemizygous germ-line deletions encompassing WT1) centered on band $11 \mathrm{p} 13$, whereas the sporadic Wilms' tumors showed a more distal epicenter of $\mathrm{LOH}$ at band 11p15 (Fig. 1). Consistent with the relative rarity of WT1 mutations in sporadic Wilms' tumors, only two of the sporadic tumors showed $\mathrm{LOH}$ restricted to band $11 \mathrm{p} 13$, and in one of these cases, we have confirmed previously the presence of a WT1 mutation (9).

Mitotic Recombination Produces $\mathrm{LOH}$ on 11p15, Whereas Reduction to Hemizygosity Produces $\mathrm{LOH}$ for Other Chromosomal Regions

$\mathrm{LOH}$ can occur via at least two different pathways: Mitotic recombination (crossing over) between the paternal and the maternal homologues of a given chromosome can produce homozygosity for alleles distal to the cross-over in one daughter cell, whose clonal progeny go on to populate the tumor. This pathway does not produce a net reduction in chromosomal copy number; rather, it leads to loss of one set of parental alleles and duplication of the other. This pathway is relevant to the $11 \mathrm{p} 15.5$ WT2 region, which contains the imprinted $I G F 2$ gene, which is active only on the paternal homologue and which therefore undergoes a 2-fold increase in functional gene dosage when there is loss of maternal and reduplication of paternal chromosome 11p15.5 DNA. Alternatively, LOH can reflect a true reduction to hemizygosity (i.e., a net loss of DNA, as occurs with unbalanced chromosomal translocations, chromosomal deletions, and mitotic nondisjunctions).
Karyotypes from standard cytogenetic analysis were available for 22 of the sporadic Wilms' tumors in our series. The results combining SNP chip and cytogenetic data indicated that LOH involving the p-arm of chromosome 11 is invariably due to mitotic recombination producing no net reduction in DNA copy number: in each of nine Wilms' tumors with 11p LOH and successful karyotyping, we observed two copies of chromosome 11 . In contrast, $\mathrm{LOH}$ for chromosome arms $7 p$ and $16 q$ typically reflected a net loss of DNA, with reduction to hemizygosity. In four successfully karyotyped tumors with $7 \mathrm{p}$ LOH by SNP chip analysis, we found physical loss of one copy of the p-arm of chromosome 7 in each case. Similarly, for two tumors with $16 \mathrm{q}$ LOH by SNP chips analysis and successful karyotyping, both showed physical loss of the q-arm of chromosome 16 resulting in hemizygosity. This net loss of DNA was attributable in some cases to the formation of unbalanced chromosomal translocations: isochromosome $7 \mathrm{q}$ and $\mathrm{t}(1 ; 16)$, respectively; however, in other cases, we observed simple deletions of the relevant chromosome arms as illustrated for a Wilms' tumor with 7 p LOH in Fig. 2.

\section{Minimal Regions of $\mathrm{LOH}$}

The boundaries of the smallest regions of recurrent $\mathrm{LOH}$ defined in the series of sporadic Wilms' tumors are shown in Fig. 3. Most of these regions are consistent with prior studies, and the $10 \mathrm{~K}$ SNP chip data highlight some additional areas that may contain tumor suppressor genes. Notably, five sporadic Wilms' tumors showed LOH encompassing chromosome 4q, and the SNP chip data, supplemented by higher resolution mapping at individual SNPs, define a novel 2.4-Mb minimal region of LOH (Figs. 3 and 4) that includes the $C X X C 4 / I D A X$ gene, encoding an inhibitor of the Wnt signaling pathway (30-32). The results of RFLP analysis and direct sequencing shown in Fig. 4 provide an independent validation of the SNP chip data by confirming $\mathrm{LOH}$ in this region. We sequenced the entire coding region of $C X X C 4$ in two of the tumors with $\mathrm{LOH}$, including the case with the minimal deletion, and did not find mutations. Southern blotting with quantitation by phosphorimaging showed $C X X C 4$ hemizygosity in these cases (Fig. 4B). This reduction in $C X X C 4$ copy number may contribute to Wilms' tumor progression, but an additional nine genes are present in the minimal region of 4q24-q25 LOH (Fig. 4A), and these remain to be examined for mutations.

Several other small minimal regions of $\mathrm{LOH}$ on other chromosomes are suggested by the data in Fig. 3. As is true for the 4q24-q25 region, due to the overall genomic stability of Wilms' tumors, many of these regions are defined by single tumor cases. In the future, pooling of data from multiple independent case series analyzed by SNP chips may allow these regions to be validated.

The Number of Chromosomes Affected by $\mathrm{LOH}$ in Sporadic Wilms' Tumors Increases with Clinical Stage at Presentation

Advanced clinical stage at presentation, particularly metastatic disease (stage IV), is an independent risk factor for treatment failure in Wilms' tumors (33), and local tumor spread (stage III) has been associated with a greater probability of local recurrence after treatment (34). Information concerning the 


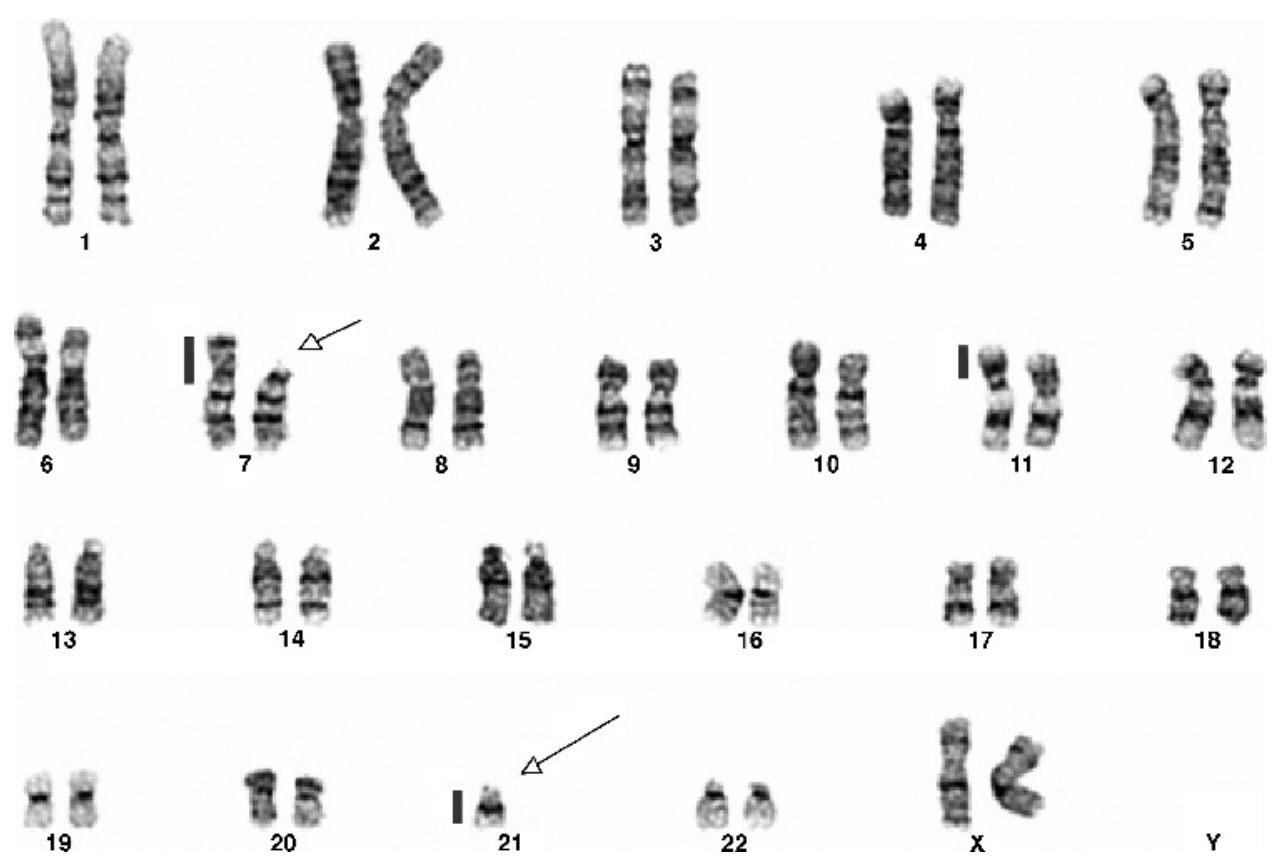

FIGURE 2. LOH in Wilms' tumors reflects mitotic recombination affecting chromosome $11 \mathrm{p} 15$ but reduction to hemizygosity affects other chromosomal regions. An example of a characteristic sporadic Wilms' tumor karyotype is shown. Arrows, regions of DNA deletion; bars, regions of LOH detected by analysis of paired tumor and normal on the SNP chips. Chromosome 11 is cytogenetically normal but showed LOH by SNP chip analysis.

clinical stage was available for 43 of the sporadic Wilms' tumors in our series. We plotted the number of chromosomal regions affected by $\mathrm{LOH}$ in each tumor as a function of stage and found that this number increased steadily with increasing stage (Fig. 5). Somewhat surprisingly, fully $60 \%$ of stage I Wilms' tumors (confined to the kidney at presentation) showed no LOH (mean value for number of regions affected by $\mathrm{LOH}$ per tumor at stage I is 1.4). In contrast, most stage IV tumors (metastatic to distant sites at presentation) contained three or more regions of $\mathrm{LOH}$ (mean, 3.9) and all of these advanced-stage tumors showed at least two regions of $\mathrm{LOH}$. Because the transition from stage I-II to stage III-IV has prognostic importance for both local recurrence and treatment failure, we analyzed these data statistically by comparing the number of chromosomal regions with $\mathrm{LOH}$ in each tumor for the stage I-II cases versus stage IIIIV and found a significant difference $(P=0.006, t$ test $)$. Combining the stage I-III cases and comparing with stage IV also revealed a significant difference $(P=0.004, t$ test $)$.

The chromosomal region individually showing the strongest statistical association of $\mathrm{LOH}$ with advanced clinical stage was $16 \mathrm{q}$ (stage I-III compared with stage IV; $P=0.037$, Fisher's exact test). Consistent with the significant overall association of chromosomal deletions with advanced clinical stage, trends for increasing $\mathrm{LOH}$ with increasing clinical stage were also seen for chromosomes 1p, 14q, and 17p (Fig. 6A), which were never lost in the stage I tumors. However, these did not reach statistical significance individually probably due to the small number of cases with $\mathrm{LOH}$ for each chromosome. LOH encompassing chromosome band $11 \mathrm{p} 15$ occurred at earlier stages, whereas loss of chromosome $7 \mathrm{p}$ did not show a discernible association with clinical stage (Fig. 6B; data not shown).
A likely target of the $17 \mathrm{p} \mathrm{LOH}$ is the TP53 tumor suppressor gene, a locus that shows a well-known association with tumor progression in other systems and that has been implicated previously as mutated in some anaplastic Wilms' tumors (35). We sequenced several of the most frequently mutated exons of TP53 (exons 5-9) in six of the Wilms' tumors with $17 \mathrm{p} \mathrm{LOH}$ and found a single case (a stage IV tumor) with a loss-of-function mutation: a 4-bp insertion in exon 5 that produced a frameshift and premature STOP codon (data not shown). The remaining cases may have TP53 mutations in other exons or mutations in another gene on this chromosome arm.

\section{Gain of DNA Methylation at the H19 5' Differentially Methylated Region Frequently Precedes Chromosomal Losses}

As shown in Fig. 6B, LOI as indicated by biallelic DNA methylation at the $H 195^{\prime}$ differentially methylated region (DMR) was more common in early-stage compared with latestage Wilms' tumors (frequency of LOI in stage I-III versus stage IV; $P=0.037$, Fisher's exact test). Strikingly, many of these early-stage Wilms' tumors with LOI entirely lacked $\mathrm{LOH}$ in the genome-wide scan. Figure 7 shows the genomewide patterns of LOH for 58 sporadic Wilms' tumors in which we determined the LOI status: of the 22 tumors with LOI, 8 showed no evidence of $\mathrm{LOH}$. Among the remaining 14 tumors with LOI, various chromosomes showed $\mathrm{LOH}$, with $11 \mathrm{q}$ affected in 6 cases, $16 \mathrm{q}$ in 4 cases, and $7 \mathrm{p}$ in 4 cases. In comparing the rates of $\mathrm{LOH}$ for these three chromosomal regions in the tumors with and without LOI, none were significantly associated with LOI (Fisher's exact test, all nonsignificant). 
These data indicate that LOI frequently precedes chromosomal losses in sporadic Wilms' tumors. We found additional evidence for this temporal sequence from analyzing mosaicism. In our prior study of chromosome 16q LOH in Wilms' tumors using conventional microsatellite markers, we have noticed that the loss of alleles on this chromosome is sometimes partial, consistent with the loss being a late-stage event that manifests as tissue mosaicism in the growing tumor (14). Consistent with this scenario, for one of the cases in that prior study, the 16q LOH only became clear when we used DNA obtained after early passages of the tumor explant, and for other cases, we also observed partial allele losses for $16 \mathrm{q}$ markers. Because this is relevant to the relative timing of the two events, we directly compared the extent of DNA methylation at the H19 5' DMR with the extent of chromosome $16 \mathrm{q}$ LOH in primary Wilms' tumors. In the tumor illustrated in Fig. 8A, the gain of DNA methylation was complete, whereas the $16 \mathrm{q} \mathrm{LOH}$ was partial, indicating that the LOI had preceded the LOH. In fact, LOI can occur as a preneoplastic lesion in Wilms' tumor patients, being detectable in the kidney parenchyma surrounding the tumor, even in the absence of histologic abnormalities, such as nephrogenic rests. We reported previously two cases of sporadic Wilms' tumors with this finding (36), and we have since found two additional examples in our expanded case series. In one of these cases, the SNP chip analysis revealed chromosome $7 \mathrm{p} \mathrm{LOH}$ in the tumor. As shown in Fig. 8B, a side-by-side analysis of $H 195^{\prime}$ DMR methylation and chromosome $7 \mathrm{p} \mathrm{LOH}$ in the tumor and adjacent kidney parenchyma from this case confirms that epigenetic lesion (LOI) occurred well before the genetic lesion (chromosomal loss) in the evolution of this tumor. As in our previously reported cases (36), nephrogenic rests were not found in standard histologic sections from this case and the patient did not meet clinical criteria for Beckwith-Wiedemann syndrome. However, the patient was in the 97th percentile for height and 95th percentile for weight, suggesting somatic overgrowth.

\section{Discussion}

Distinct Pathways of Wilms' Tumor Development in Sporadic versus Syndromic Cases

Work from several laboratories, including ours, has led to the realization that the genetic and epigenetic events in the formation of sporadic versus syndromic (Denys-Drash and WAGR) Wilms' tumors are largely distinct. First, WT1 lossof-function mutations are invariably present in the syndromic tumors and are rare in the sporadic tumors (8-10). Second, $\beta$-catenin $(C T N N B 1)$ gain-of-function mutations are common in the syndromic tumors and, among the sporadic tumors, are only found when a WT1 mutation is also present $(9,37)$. Third, syndromic Wilms' tumors, together with the rare sporadic cases that have WT1 mutations, show a characteristic gene expression signature that differs from that of the common (WT1 wild-type/CTNNB1 wild-type) sporadic cases (9). Fourth, Wilms' tumors occurring in Denys-Drash and WAGR syndrome showed a lower frequency of IGF2 LOI compared with sporadic Wilms' tumors (38), a finding that is substantiated by our current data. Most recently, Ruteshouser et al. reported a genome scan similar in design to ours, which uncovered distinct patterns of $\mathrm{LOH}$ in syndromic versus sporadic Wilms' tumors (39). Our current data comparing syndromic and sporadic Wilms' tumors are consistent with

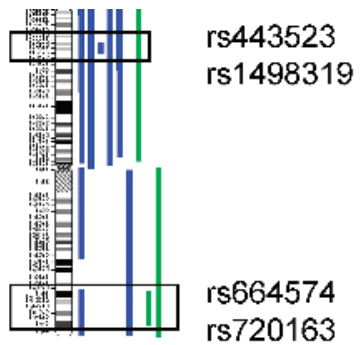

Chr 1

rs720163

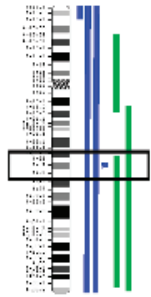

Chr 4 rs233973 rs 17036613

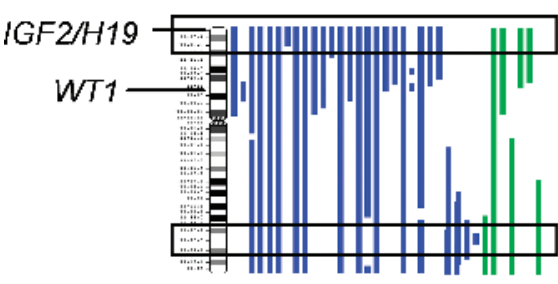

pter rs2896587

rs 10488743 rs496547

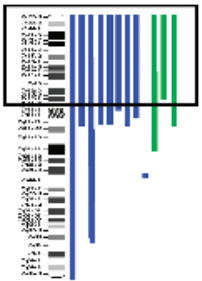

Chr 7 pter

rs 1574717

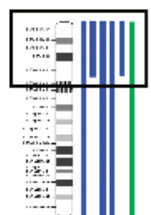

pter

rs953113

Chr 17

\section{Chr 11}

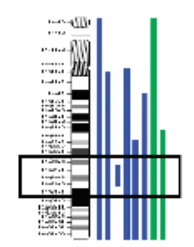

rs755212 rs961097

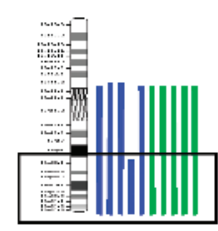

rs909003

qter

\section{Chr 14}

\section{Chr 16}

FIGURE 3. Minimal regions of recurrent LOH in sporadic Wilms' tumors. The boundaries of all regions showing LOH in four or more tumors are indicated by dbSNP ID numbers. The bars indicating LOH are color coded as in Fig. 1. 
A

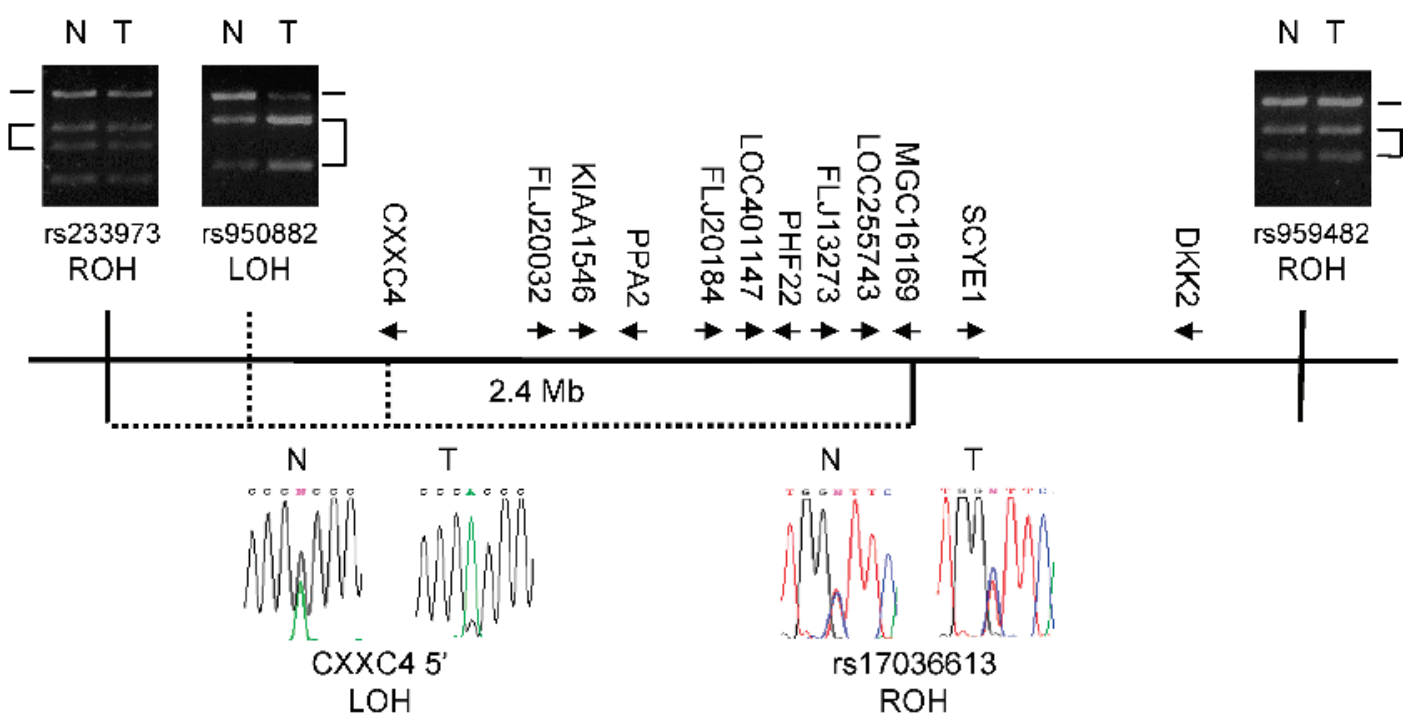

B
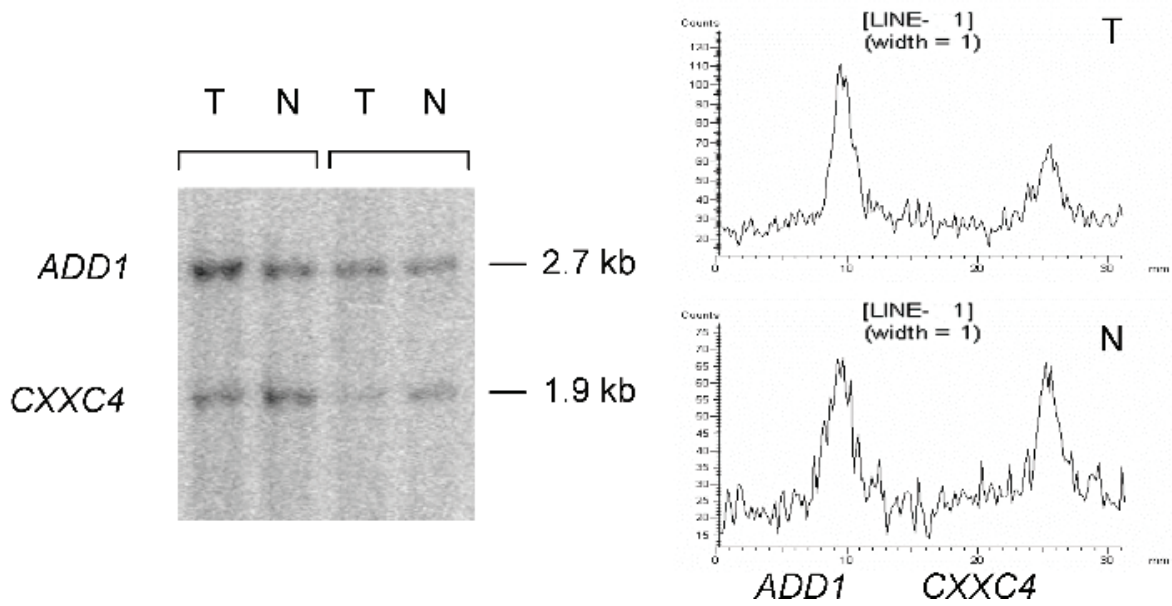

FIGURE 4. Validation of the SNP chip data for the minimal region of LOH on chromosome 4q24-q25. A. DNA sequencing and RFLP analysis of five SNPs defining the minimal region of LOH (the same region of chromosome band 4q24-q25 as in Fig. 3). SNPs rs233973 and rs17036613 show retention of heterozygosity and define the borders of the region. Arrows, direction of transcription of named genes in this region. B. Reduction to hemizygosity shown by quantitative Southern blot analysis using simultaneous hybridization with ADD1 (chromosome 4p16.3) and CXXC4 (chromosome 4q24-q25) probes. The corresponding signals of the two bands from phosphorimaging are shown (right). Paired samples from two cases with LOH encompassing chromosome band 4q24-q25 are indicated: N, normal kidney; T, Wilms' tumor.

these prior reports: we found no evidence of LOI in any of the nine syndromic Wilms' tumors in our series and found a different pattern of $\mathrm{LOH}$ in these cases compared with our series of sporadic tumors.

\section{Regions of Recurrent LOH in Sporadic Wilms' Tumors}

Our findings highlight known and novel regions of $\mathrm{LOH}$ in sporadic Wilms' tumors. For the known regions of recurrent loss, including chromosome 1p, 7p, 11p, 11q, 14q, and 16q, the minimal regions of $\mathrm{LOH}$ remain large. But included in our data are several smaller regions of recurrent $\mathrm{LOH}$, notably a $2.4-\mathrm{Mb}$ region on chromosome 4q24-q25. This region of DNA contains several interesting genes, including the $C X X C 4$ gene encoding a Wnt pathway inhibitor. We did not find mutations in this gene, but quantitation Southern blot analysis indicated CXXC4 hemizygosity in the tumors with $\mathrm{LOH}$, and it is possible that a reduction in $C X X C 4$ gene dosage might contribute to sporadic Wilms' tumor progression. Alternatively, one of the other genes in the region could be the relevant target of loss. The NPNT/ LOC255743 gene, encoding an apparent orthologue of the mouse nephronectin gene, is another interesting candidate tumor suppressor, as nephronectin is an extracellular matrix component that is a ligand for $\alpha_{8} / \beta_{1}$ integrin, which is in turn a molecule that is essential for metanephric kidney maturation (40-44).

Ruteshouser et al. reported previously a restricted region of chromosome 9q LOH in Wilms' tumors (39). In our series, four sporadic tumors showed 9q LOH (Fig. 1). The regions of LOH in two of our cases do not overlap with each other or with the minimal region defined by Ruteshouser et al., whereas the two other cases showed large segments of $\mathrm{LOH}$ that do include their minimal region. Analysis of additional cases will be needed to define the molecular anatomy of chromosome 9q in Wilms' tumors. 


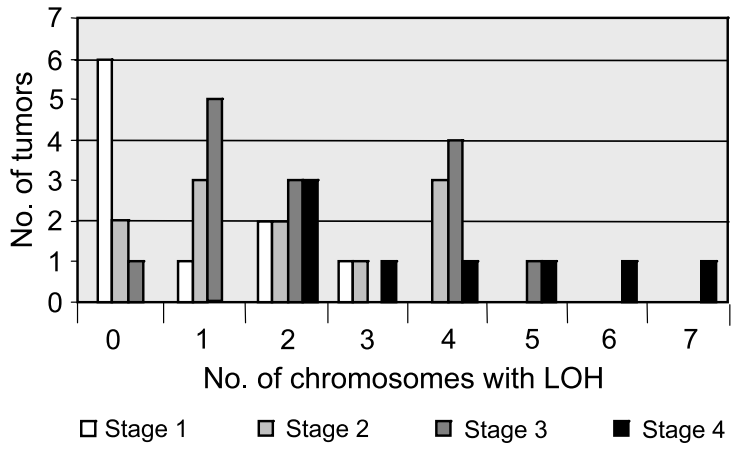

FIGURE 5. Number of chromosomal regions affected by $\mathrm{LOH}$ according to clinical stage in sporadic Wilms' tumors at presentation. Advanced-stage Wilms' tumors accumulate multiple regions of LOH. Most stage I Wilms' tumors contain no LOH regions, whereas no stage IV tumors contain fewer than two $\mathrm{LOH}$ regions.

\section{Stage Dependence and Timing of Genetic and Epige- netic Events in Sporadic Wilms' Tumors}

Our study goes beyond prior work in providing a comprehensive analysis of both genome-wide LOH and LOI in a large series of Wilms' tumors. The results, showing LOI more frequent in early-stage tumors and $\mathrm{LOH}$, particularly for chromosome arms 1p, 14q, 16q, and 17p, more frequent in latestage tumors, have implications for the timing and mechanism of LOI. The data show that LOI is an early event, often occurring in the complete absence of LOH. This finding is consistent with two prior observations. First, gain of methylation in $H 19$ upstream DNA sequences (as well as in the transcribed region of the $H 19$ gene), which is the DNA counterpart of LOI that is scored in this and many other studies, can be seen in the nonneoplastic kidney parenchyma of a substantial group of sporadic Wilms' tumor patients $(36,45)$. Second, IGF2 LOI associated with gain of methylation at the $H 195^{\prime} \mathrm{DMR}$ in various somatic tissues is the defining feature of the class of Beckwith-Wiedemann syndrome that is associated with a predisposition to Wilms' tumor $(12,46,47)$. Recently, it has been suggested that rare cases of sporadic Wilms' tumor might have LOI secondary to LOH affecting the chromosome 16q CTCF gene (48). Our data indicating that LOI usually precedes chromosome 16q LOH and is not statistically associated with loss of this chromosome arm do not provide positive support for this scenario. However, the CTCF protein plays an important role as an insulator binding protein at the $H 195^{\prime} \mathrm{DMR}$, and our results do not rule out the possibility of rare Wilms' tumors in which CTCF hemizygosity may contribute to LOI.

The fact that sporadic Wilms' tumors with LOI that present at early clinical stages often show no $\mathrm{LOH}$ raises interesting questions about the molecular pathogenesis of these cancers. Stage I Wilms' tumors, although confined to the kidney in which they arise, are nonetheless large neoplasms, which often come to clinical attention based on effects from the tumor mass. As the research community makes plans for large-scale cancer genome and cancer epigenome projects, such tumors may offer the opportunity to answer questions about the relative requirements for genetic versus epigenetic events in the early stages of tumor formation. Lastly, our finding that LOI is less common in
Wilms' tumors that are metastatic at clinical presentation compared with cases in which the tumor presents at an earlier clinical stage suggests that the tumors with LOI may be biologically different from those with $\mathrm{LOH}$.

\section{Materials and Methods}

Tumor Samples and DNA/RNA Preparation

Biopsies of 62 sporadic and 9 syndromic Wilms' tumor and, when available, adjacent normal kidney tissue were obtained with institutional review board approval. Of the sporadic Wilms' tumors, 10 were stage I, 12 were stage II, 14 were stage III, and 8 were stage IV. Stage information was not available for the remaining 18 sporadic tumors. DNA was extracted by proteinase K-SDS lysis followed by phenol-chloroform extraction and ethanol precipitation. DNA quality was assessed by gel electrophoresis and ethidium bromide staining, and only samples with intact high molecular weight DNA were used for preparing SNP chip probes. RNA was prepared from tissue pulverized under liquid nitrogen and lysed in Trizol reagent (Invitrogen, Carlsbad, CA).
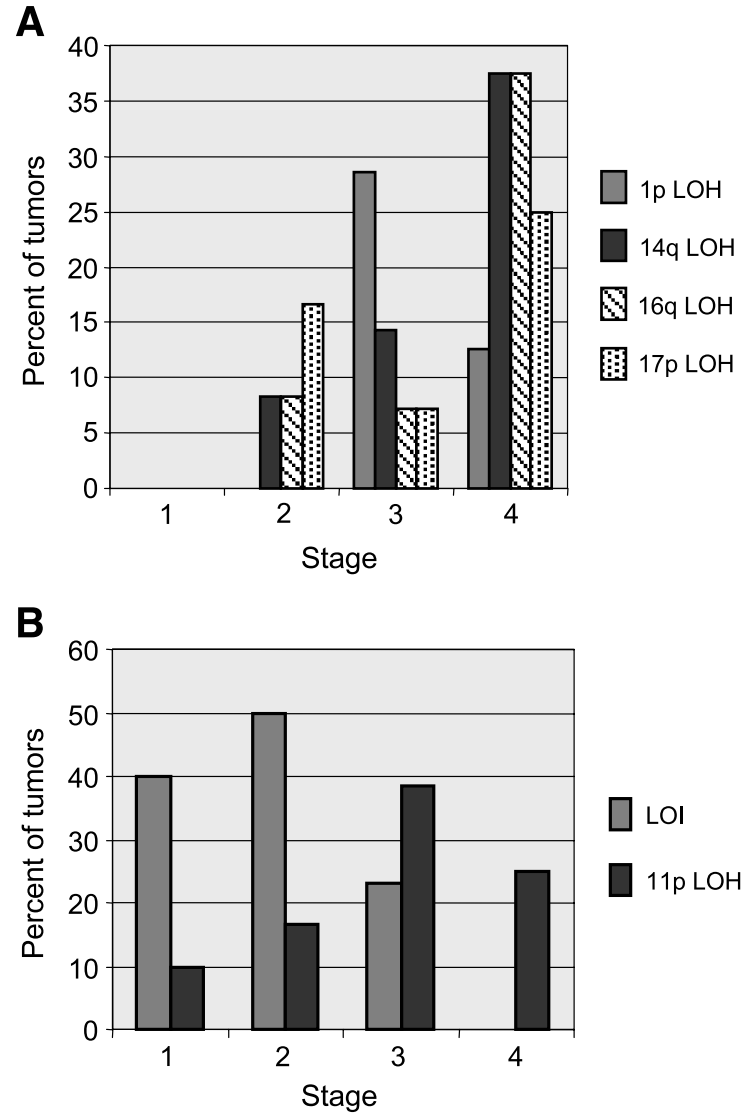

FIGURE 6. Correlations of LOH and LOI with clinical stage in sporadic Wilms' tumors. A. Data for chromosome arms 1p, 14q, 16q, and 17p. B. Data for chromosome band 11 p15 and IGF2/H19 LOI (assessed based on biallelic methylation at the $\mathrm{H}_{19} \mathrm{5}^{\prime} \mathrm{DMR}$ ). In this series of tumors, the only chromosomal region affected by $\mathrm{LOH}$ at stage I is band $11 \mathrm{p} 15$. LOI accounts for another large group of tumors at stage I. In contrast, $\mathrm{LOH}$ for the other frequently affected chromosomal regions accumulates only at higher clinical stages. 


\section{Cytogenetics}

A portion of the tumor was placed in MEM immediately after surgery for cytogenetic analysis. Tumor tissues were minced using scalpel blade and digested with 200 units $/ \mathrm{mL}$ collagenase in complete DMEM for 2 to 12 hours. Dissociated cells were washed twice with DMEM and cultures were set up in complete DMEM supplemented with insulin-transferrin-sodium selenate (Invitrogen). Tumor cells grown for 2 to 10 days were harvested for metaphase preparation, g-banded, and analyzed by standard procedures (14).

\section{Probe Preparation and SNP Chip Hybridization}

Probes were prepared from the genomic DNA samples according to the manufacturer's instructions (Affymetrix GeneChip Mapping Assay). Where necessary, DNA was concentrated using sodium acetate extraction before digesting with $X b a \mathrm{I}$. To generate the required $20 \mu \mathrm{g}$ DNA, five (instead of four) PCR reactions were set up. PCR product was concentrated using either QIAquick PCR Purification kit or MiniElute UF PCR Purification kit (Qiagen, Hilden, Germany).

\section{Analysis of SNP Chip Data}

Alleles (AA, AB, or $\mathrm{BB}$ ) at each SNP were called using the algorithm of Liu et al. (49) as implemented in the Affymetrix software package GDAS, and the data displayed in a spreadsheet sorted by chromosome and nucleotide position using Build 34 of the human genome. For normal/tumor pairs, $\mathrm{LOH}$ at a given SNP was identified as an AB-to-AA or ABto-BB conversion. For unpaired tumor samples, regional $\mathrm{LOH}$ was scored whenever an uninterrupted contiguous stretch of $>100$ homozygous (AA or BB) genotypes was encountered (see Results).

\section{Southern Blotting and Northern Blotting}

We analyzed $\mathrm{CpG}$ methylation in the H19 5' DMR by Southern blotting after digestion of genomic DNA with methylation-sensitive restriction enzymes as described previously (14). For measuring DNA copy number at the CXXC4 gene, genomic DNA from Wilms' tumors and matched nonneoplastic kidney parenchyma was digested with RsaI and the Southern blot was hybridized simultaneously with ${ }^{32} \mathrm{P}$-labeled probes for $C X X C 4$ intron 3 (intron 1 in published sequence) on chromosome arm 4q and intron 1 of the $A D D l$ gene on

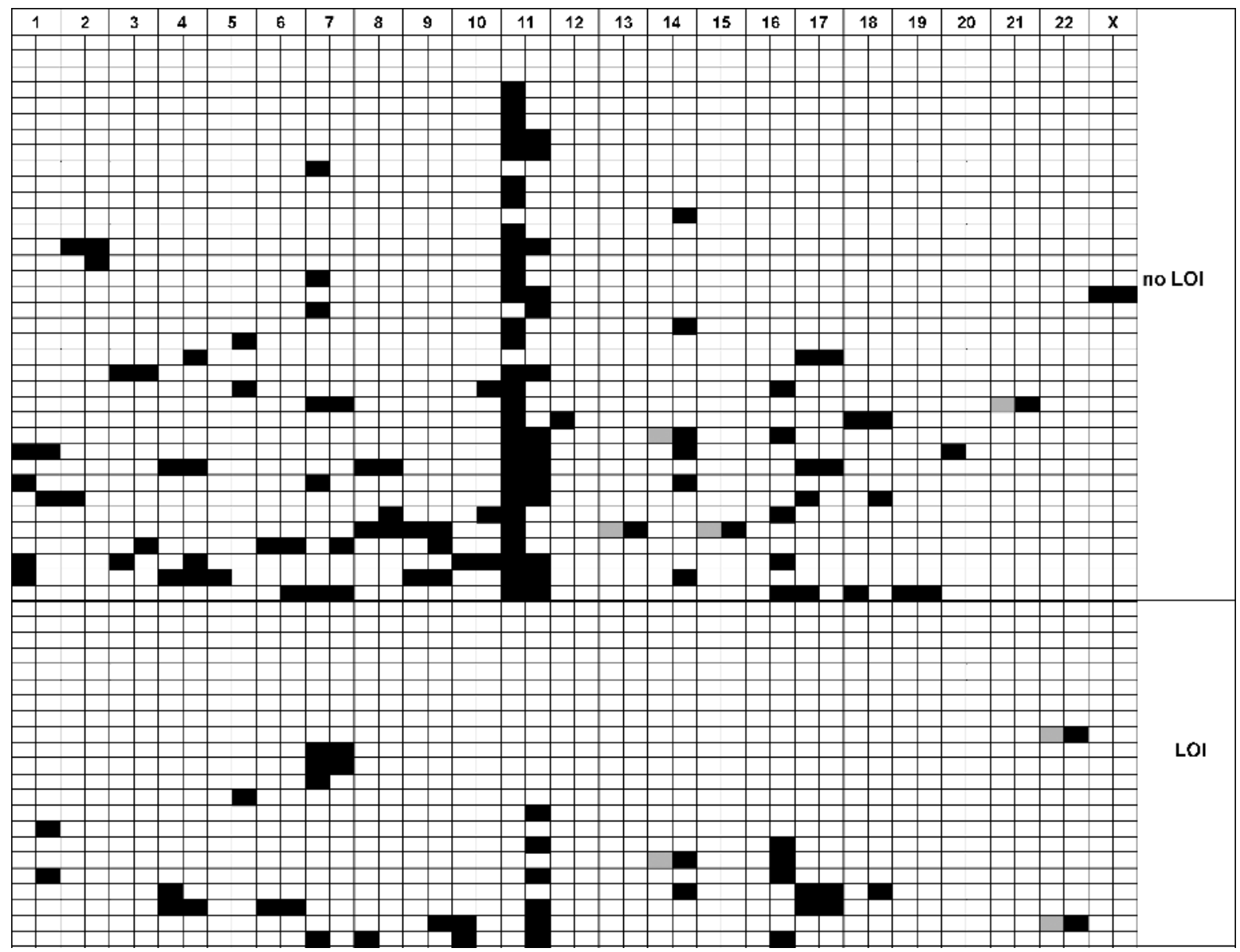

FIGURE 7. Genome-wide LOH analysis in sporadic Wilms' tumors with and without LOI. Chromosomes are numbered at the top, with the p-and q-arms represented by the left and right columns. Black rectangles, $\mathrm{LOH}$; the $\mathrm{p}$-arms of the acrocentric chromosomes were not queried by the SNP chips (gray). Wilms' tumors with LOI have a lower overall frequency of LOH than those without LOI, and no region of LOH is specifically associated with LOI. 
A

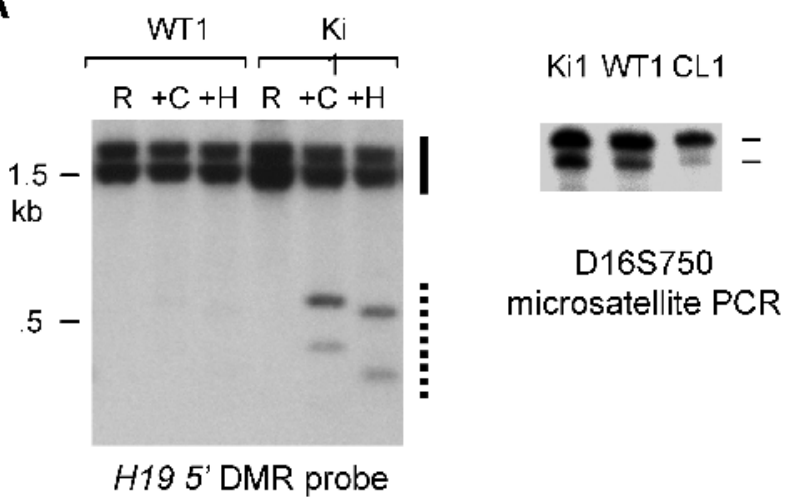

B

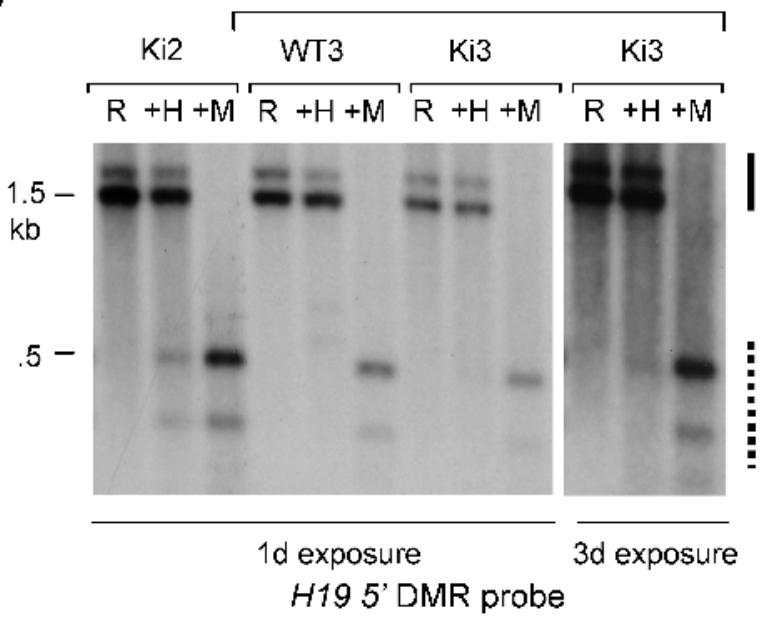

$\mathrm{Ki} 3 \mathrm{WT} 3$

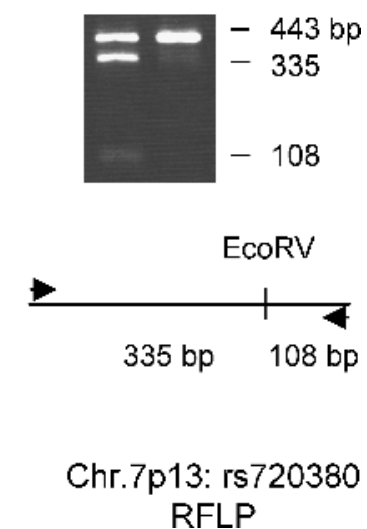

FIGURE 8. Timing of gain of methylation at the H19 $5^{\prime}$ DMR relative to LOH at $16 q$ and $7 p$ in Wilms' tumors. A. Gain of methylation at the H19 $5^{\prime}$ DMR preceding LOH for chromosome 16q. DNA methylation of the H19 $5^{\prime}$ DMR was assessed by Southern blotting (left). Biallelic DNA methylation is visualized as complete protection from digestion by the methylation-sensitive enzymes Cfol and Hpall in the tumor DNA, seen as absence of the low molecular weight restriction fragments (dashed line) and relatively increased intensity of the high molecular weight intact $R$ sal fragments (solid line). $\mathrm{R}$, Rsal; $\mathrm{C}$, Cfol, $\mathrm{H}$, Hpall. See ref. 14 for a map of the H19 DMR probe. Mosaicism for LOH at a microsatellite marker on band 16q23.1 is observed in the same primary Wilms' tumor DNA with partial loss of the lower band, whereas there is nearly complete LOH in the tissue culture explant of this tumor after three passages (right). WT1, Wilms' tumor; Ki1, corresponding normal kidney; CL1, tumor explant. B. Gain of methylation at the H19 5' DMR preceding LOH for chromosome 7p. In this case, the Wilms' tumor (WT3) shows both gain of methylation at the H19 $5^{\prime}$ DMR as indicated by Southern blotting (left) and LOH for chromosome $7 \mathrm{p}$ as indicated by the SNP chip data and confirmed by PCR/RFLP analysis (right). The nonneoplastic kidney parenchyma from this case (Ki3) shows substantial gain of methylation at the H19 5' DMR (left) but does not show LOH for chromosome 7p (right). For comparison, a kidney from a different Wilms' tumor patient (Ki2) with an essentially normal pattern of monoallelic H19 $5^{\prime}$ DMR methylation is included (left). Mspl restriction enzyme (non-methylation-sensitive isoschizomer of Hpall) was included in this Southern analysis to show the band pattern from complete digestion. R, Rsal; H, Hpall; M, Mspl.

chromosome arm 4p. Probes were generated by genomic PCR with $C X X C 4$ PCR primers: CCATATGGACTTGACTTAGTGC and CCTAGCAAAGGTGAACACCTG and ADD1 PCR primers: AATATCATGTAACTACAGAGACTC and GCAAGAAGAATCACGGAGCGT. Northern blots were done after electrophoresis of $6 \mu \mathrm{g}$ denatured total RNA through 1\% agarose gels containing formaldehyde.

\section{DNA Sequencing and RFLP Analysis}

For analyzing $\mathrm{LOH}$ in the minimal region of $\mathrm{LOH}$ on chromosome 4q, genomic PCR primers were rs233973-US (TGGTGGTGGTAAGGCAGAGC) and rs233973-DS (GGTGGGTGGTGTTTCAGTCG), PCR product digested with Tsp45I; rs950882-US (ATGATGCAGGCCAAGGAGCT) and rs950882-DS (GTAAGTTCCACAGCGTAGGCA), PCR product digested with $B c c \mathrm{I}$; and rs959482-US (ACTCT-
GAGGCCAAACTCCTTG) and rs959482-DS (CAGGGTTCTCCCCATTCAGC), PCR product digested with Psi I. CXXC4 intron 1 SNP (identified by us; rs number not assigned) CXXC4-intron1-US (GGTGTCCACGTCCCTGCGT) and CXXC4-intron1-DS (TTCACAATCCCAACGGACAGC), PCR product directly sequenced; rs17036613-US (ATATAATGTTCTTGGAGTACTGTG) and rs17036613-DS (CTTGTTCAGATATGCACTATGAC), PCR product directly sequenced. Primer sequences for amplifying and sequencing the coding region of $C X X C 4$ are available on request. Exons 5 to 9 of the TP53 gene were sequenced after genomic PCR using flanking intronic primers; primer sequences are available on request. The RFLP for chromosome 7p was assessed by genomic PCR using primers rs720380-F1 (AGTTGGCTAGACCATGACACC) and rs720380-R1 (TTCCTCTAGAACCATCCTTGG) followed by Eco RV digestion. 


\section{References}

1. Knudson AG, Jr., Strong LC. Mutation and cancer: a model for Wilms' tumor of the kidney. J Natl Cancer Inst 1972;48:313-24.

2. Riccardi VM, Sujansky E, Smith AC, Francke U. Chromosomal imbalance in the Aniridia-Wilms' tumor association: $11 \mathrm{p}$ interstitial deletion. Pediatrics 1978; 61:604-10

3. Francke U, Holmes LB, Atkins L, Riccardi VM. Aniridia-Wilms' tumor association: evidence for specific deletion of $11 \mathrm{p} 13$. Cytogenet Cell Genet 1979; $24: 185-92$

4. Kaneko Y, Egues MC, Rowley JD. Interstitial deletion of short arm of chromosome 11 limited to Wilms' tumor cells in a patient without aniridia. Cancer Res 1981;41:4577-8.

5. Call KM, Glaser T, Ito CY, et al. Isolation and characterization of a zinc finger polypeptide gene at the human chromosome 11 Wilms' tumor locus. Cell 1990; 60:509-20

6. Gessler M, Poustka A, Cavenee W, Neve RL, Orkin SH, Bruns GA Homozygous deletion in Wilms tumours of a zinc-finger gene identified by chromosome jumping. Nature 1990;343:774-8.

7. Haber DA, Buckler AJ, Glaser T, et al. An internal deletion within an $11 \mathrm{p} 13$ zinc finger gene contributes to the development of Wilms' tumor. Cell 1990;61: $1257-69$.

8. Gessler M, Konig A, Arden K, et al. Infrequent mutation of the WT1 gene in 77 Wilms' Tumors. Hum Mutat 1994;3:212-22.

9. Li CM, Kim CE, Margolin AA, et al. CTNNB1 mutations and overexpression of Wnt/B-catenin target genes in WT1-mutant Wilms' tumors. Am J Pathol 2004; $165: 1943-53$.

10. Little M, Wells C. A clinical overview of WT1 gene mutations. Hum Mutat 1997;9:209-25

11. Feinberg AP, Tycko B. The history of cancer epigenetics. Nat Rev Cancer 2004; $4: 143-53$

12. Bliek J, Gicquel C, Maas S, Gaston V, Le Bouc Y, Mannens M. Epigenotyping as a tool for the prediction of tumor risk and tumor type in patients with BeckwithWiedemann syndrome (BWS). J Pediatr 2004;145:796-9.

13. Sparago A, Cerrato F, Vernucci M, Ferrero GB, Silengo MC, Riccio A Microdeletions in the human H19 DMR result in loss of IGF2 imprinting and Beckwith-Wiedemann syndrome. Nat Genet 2004;36:958-60

14. Yeh A, Wei M, Golub SB, Yamashiro DJ, Murty VV, Tycko B. Chromosome arm $16 \mathrm{q}$ in Wilms tumors: unbalanced chromosomal translocations, loss of heterozygosity, and assessment of the CTCF gene. Genes Chromosomes Cancer 2002:35:156-63.

15. Grundy PE, Telzerow PE, Breslow N, Moksness J, Huff V, Paterson MC. Loss of heterozygosity for chromosomes $16 \mathrm{q}$ and $1 \mathrm{p}$ in Wilms' tumors predicts an adverse outcome. Cancer Res 1994;54:2331-3.

16. Solis V, Pritchard J, Cowell JK. Cytogenetic changes in Wilms' tumors. Cancer Genet Cytogenet 1988;34:223-34.

17. Sandoval C, Stringel G, Ozkaynak MF, Tugal O, Jayabose S. Isochromosome 7q and Wilms tumor. Cancer Genet Cytogenet 1998;104:61-5.

18. Rubin BP, Pins MR, Nielsen GP, et al. Isochromosome $7 \mathrm{q}$ in adult Wilms' tumors: diagnostic and pathogenetic implications. Am J Surg Pathol 2000; $24: 1663-9$.

19. Powlesland RM, Charles AK, Malik KT, et al. Loss of heterozygosity at $7 \mathrm{p}$ in Wilms' tumour development. Br J Cancer 2000;82:323-9.

20. Peier AM, Meloni AM, Erling MA, Sandberg AA. Involvement of chromosome 7 in Wilms tumor. Cancer Genet Cytogenet 1995;79:92-4

21. Fletcher JA, Renshaw AA. Isochromosome 7q in adult Wilms' tumor. Cancer Genet Cytogenet 1996;86:168-9.

22. Peres EM, Savasan S, Cushing B, Abella S, Mohamed AN. Chromosome analyses of 16 cases of Wilms tumor: different pattern in unfavorable histology. Cancer Genet Cytogenet 2004;148:66-70.

23. Dao D, Walsh CP, Yuan L, et al. Multipoint analysis of human chromosome 11p15/mouse distal chromosome 7: inclusion of H19/IGF2 in the minimal WT2 region, gene specificity of H19 silencing in Wilms' tumorigenesis and methylation hyper-dependence of H19 imprinting. Hum Mol Genet 1999;8:1337-52.

24. Coppes MJ, Bonetta L, Huang A, et al. Loss of heterozygosity mapping in Wilms tumor indicates the involvement of three distinct regions and a limited role for nondisjunction or mitotic recombination. Genes Chromosomes Cancer 1992; $5: 326-34$.
25. Mannens M, Devilee P, Bliek J, et al. Loss of heterozygosity in Wilms' tumors, studied for six putative tumor suppressor regions, is limited to chromosome 11. Cancer Res 1990;50:3279-83.

26. Slater RM, de Kraker J, Voute PA, Delemarre JF. A cytogenetic study of Wilms' tumor. Cancer Genet Cytogenet 1985;14:95-109.

27. Sheng WW, Soukup S, Bove K, Gotwals B, Lampkin B. Chromosome analysis of 31 Wilms' tumors. Cancer Res 1990;50:2786-93.

28. Kaneko Y, Homma C, Maseki N, Sakurai M, Hata J. Correlation of chromosome abnormalities with histological and clinical features in Wilms' and other childhood renal tumors. Cancer Res 1991;51:5937-42.

29. Hoglund M, Gisselsson D, Hansen GB, Mitelman F. Wilms tumors develop through two distinct karyotypic pathways. Cancer Genet Cytogenet 2004;150:9-15. 30. Hino S, Kishida S, Michiue T, et al. Inhibition of the Wnt signaling pathway by Idax, a novel Dvl-binding protein. Mol Cell Biol 2001;21:330-42.

31. London TB, Lee HJ, Shao Y, Zheng J. Interaction between the internal motif KTXXXI of Idax and mDvl PDZ domain. Biochem Biophys Res Commun 2004; $322 \cdot 326-32$

32. Michiue T, Fukui A, Yukita A, et al. XIdax, an inhibitor of the canonical Wnt pathway, is required for anterior neural structure formation in Xenopus. Dev Dyn 2004:230:79-90

33. Weirich A, Ludwig R, Graf N, et al. Survival in nephroblastoma treated according to the trial and study SIOP-9/GPOH with respect to relapse and morbidity. Ann Oncol 2004;15:808-20.

34. Shamberger RC, Guthrie KA, Ritchey ML, et al. Surgery-related factors and local recurrence of Wilms tumor in National Wilms Tumor Study 4. Ann Surg 1999;229:292-7.

35. Bardeesy N, Falkoff D, Petruzzi MJ, et al. Anaplastic Wilms' tumour, a subtype displaying poor prognosis, harbours p53 gene mutations. Nat Genet 1994; $7: 91-7$.

36. Moulton T, Crenshaw T, Hao Y, et al. Epigenetic lesions at the H19 locus in Wilms' tumour patients. Nat Genet 1994;7:440-7.

37. Maiti S, Alam R, Amos CI, Huff V. Frequent association of $\beta$-catenin and WT1 mutations in Wilms tumors. Cancer Res 2000;60:6288-92.

38. Ravenel JD, Broman KW, Perlman EJ, et al. Loss of imprinting of insulinlike growth factor-II (IGF2) gene in distinguishing specific biologic subtypes of Wilms tumor. J Natl Cancer Inst 2001;93:1698-703.

39. Ruteshouser EC, Hendrickson BW, Colella S, Krahe R, Pinto L, Huff V. Genome-wide loss of heterozygosity analysis of WT1-wild-type and WT1-mutant Wilms tumors. Genes Chromosomes Cancer 2005;43:172-80.

40. Brandenberger R, Schmidt A, Linton J, et al. Identification and characterization of a novel extracellular matrix protein nephronectin that is associated with integrin $\alpha_{8} \beta_{1}$ in the embryonic kidney. J Cell Biol 2001;154:447-58.

41. Huang JT, Lee V. Identification and characterization of a novel human nephronectin gene in silico. Int J Mol Med 2005;15:719-24.

42. Valerius MT, Patterson LT, Feng Y, Potter SS. Hoxa 11 is upstream of integrin $\alpha_{8}$ expression in the developing kidney. Proc Natl Acad Sci U S A 2002; 99:8090-5

43. Hartner A, Haas C, Amann K, Sterzel RB. Aspects of the renal phenotype of adult $\alpha_{8}$ integrin-deficient mice. Nephrol Dial Transplant 2002;17 Suppl 9: $71-2$.

44. Muller U, Wang D, Denda S, Meneses JJ, Pedersen RA, Reichardt LF. Integrin $\alpha_{8} \beta_{1}$ is critically important for epithelial-mesenchymal interactions during kidney morphogenesis. Cell 1997;88:603-13

45. Okamoto K, Morison IM, Taniguchi T, Reeve AE. Epigenetic changes at the insulin-like growth factor II/H19 locus in developing kidney is an early event in Wilms tumorigenesis. Proc Natl Acad Sci U S A 1997;94:5367-71.

46. DeBaun MR, Niemitz EL, McNeil DE, Brandenburg SA, Lee MP, Feinberg AP. Epigenetic alterations of H19 and LIT1 distinguish patients with BeckwithWiedemann syndrome with cancer and birth defects. Am J Hum Genet 2002; 70:604-11.

47. Engel JR, Smallwood A, Harper A, et al. Epigenotype-phenotype correlations in Beckwith-Wiedemann syndrome. J Med Genet 2000;37:921 -6.

48. Mummert SK, Lobanenkov VA, Feinberg AP. Association of chromosome arm 16q loss with loss of imprinting of insulin-like growth factor-II in Wilms tumor. Genes Chromosomes Cancer 2005;43:155-61.

49. Liu WM, Di X, Yang G, et al. Algorithms for large-scale genotyping microarrays. Bioinformatics 2003;19:2397-403. 